\title{
Hot new therapy for sepsis and the acute respiratory distress syndrome
}

\author{
Arthur S. Slutsky \\ Department of Medicine, and Department of Critical Care Medicine, St. Michael's Hospital, Toronto, Ontario, Canada \\ Interdepartmental Division of Critical Care Medicine, University of Toronto, Toronto, Ontario, Canada \\ J. Clin. Invest. 110:737-739 (2002). doi:10.1172/JCI200216551.
}

In 1962 Ritossa demonstrated that exposing Drosophila to elevations of temperature produced "puffing" patterns of polytene chromosomes indicating increased gene expression (1). Five years later, Ashbaugh and colleagues described a new clinical syndrome, a form of non-cardiogenic pulmonary edema that they called the acute respiratory distress syndrome (ARDS) (2). These unrelated papers from widely disparate research areas pioneered independent research fields which are now intersecting, as demonstrated by the article by Weiss and colleagues in this issue of the JCI (3). This convergence of basic science with clinical research promises to influence the care of our most critically ill patients and offers an excellent model of how basic scientific discoveries, without any apparent clinical implications, can lead to novel therapies. Here, I will focus on the implications of these findings for possible new therapeutic options for ARDS, as well as on how this study provides new insights into the pathophysiology of sepsis/ARDS.

\section{The heat shock (stress) response} Approximately 10 years after Ritossa's discovery, Tissières and colleagues

\footnotetext{
Address correspondence to: Arthur S. Slutsky, St. Michael's Hospital, Room 4-042 Queen Wing, 30 Bond Street, Toronto, Ontario M5B 1W8, Canada.

Phone: (416) 864-5637; Fax: (416) 864-5117; E-mail: arthur.slutsky@utoronto.ca.

Conflict of interest: No conflict of interest has been declared.

Nonstandard abbreviations used: acute respiratory distress syndrome (ARDS); heat shock protein (HSP); heat shock factor (HSF); multiple organ dysfunction syndrome (MODS).
}

demonstrated that the "puffing" pattern observed in Drosophila salivary glands reflects the upregulation of genes encoding heat shock proteins (HSPs) (4). This heat shock response, now commonly referred to as the stress response, consists of the transcription and translation of a set of HSPs, highly conserved proteins ranging from 8-110 kDa that are classified into families based roughly on the molecular mass of a typical member $(5-7)$. The best characterized stress protein family is HSP-70, which is found in inducible and constitutive forms in many cells. The two major isoforms of this family are the constitutively expressed HSP-73 and the stress-inducible HSP-72. The latter has been widely studied and is thought to play a major role in the cytoprotection induced by the stress response. For this reason, HSP-72 was used by Weiss et al. in their study (3).

The potential importance of the stress response is demonstrated by the fact that it is ubiquitous in nature, occurs in virtually all organisms from prokaryotes to humans, and by the observation that there is tremendous homology among these proteins across virtually all living cells. Although the precise function of the stress proteins is not known, it is clear from a number of studies that they have cytoprotective effects. The first such effect that was described was that of thermotolerance: heating cells to a few degrees Celsius above their resting temperature for just a few minutes confers protection a few hours later to a heat stimulus that would otherwise be lethal. A number of elegant studies have shown that this protection can be related to specific heat shock proteins.
For example, Riabowol and colleagues inserted monoclonal antibodies to HSP-70 protein into cells and showed that they lost their thermotolerance compare with cells that had control antibodies injected (8). Another important feature of this response is cross-tolerance: heating cells can induce tolerance to other noxious stimuli such as oxidant stress, and conversely, induction of the stress response by nonthermal means can induce thermotolerance. The mechanism mediating these effects appears to be related to the chaperone function of the heat shock proteins, which appears to protect nascent proteins from denaturation (9).

The mechanisms by which the stress response is induced are not known with certainty but are thought to relate to the ability of denaturated proteins in the cytoplasm to stimulate the trimerization of heat shock factor (HSF). This protein can then translocate to the nucleus and interact with heat shock elements in the promoters of HSP70 and other target genes. A large number of different stimuli may initiate HSF trimerization and translocation, including drugs (e.g., d-lysergic acid diethylamide [LSD], salicylates), transition metals, chemicals (e.g., ethanol, sodium arsenite), and clinically relevant stresses like anoxia, ischemia, oxidant stress, and shock (10). The latter stimuli are particularly important since they are commonly observed in patients and are common in the critical care setting.

\section{The acute respiratory distress syndrome (ARDS)}

ARDS is a clinical syndrome of noncardiogenic pulmonary edema associated with pulmonary infiltrates, stiff 
lungs, and severe hypoxemia (11). ARDS is an inflammatory disease characterized by abnormalities in the coagulation system and an imbalance of pro- and anti-inflammatory compounds such as cytokines. The pathology of ARDS involves hyaline membranes, endothelial and epithelial injury, loss of epithelial integrity, and increased alveolar-capillary permeability. Classically, this loss of alveolar-capillary integrity has been thought to be paramount in producing the clinical manifestations of ARDS by increasing fluid flux into the alveoli. However, as discussed below, this increased leakiness can also lead to loss of lung compartmentalization, with the result that inflammatory mediators from the lung can enter the circulation and induce systemic consequences.

ARDS affects about 50 per 100,00 population and leads to the demise of $-30-50 \%$ of affected patients. There is some evidence that mortality has been decreasing over the past decade (12) but, despite intense research for over 30 years, no specific therapies have been shown in large-scale human trials to be effective (13). The only approach that has been shown to have an impact on mortality is the use of a ventilatory strategy that reduces iatrogenic lung injury (14).

It is within this context of a complex, lethal disease, with no effective therapy, that we should view the study by Weiss and colleagues. A number of previous studies had demonstrated that induction of the stress response by heating or by non-thermal approaches can mitigate experimental acute lung injury and sepsis (15-19). However, in all of these studies, the stress response was produced by heating the animals or by using sodium arsenite, approaches that are not readily amenable to clinical application. Moreover, because these treatments provoked a full systemic stress response, such studies did not reveal a precise mechanism of protection. By contrast, Weiss and colleagues administered a recombinant E1,E3deleted adenovirus expressing HSP-70 (AdHSP-70) directly into the trachea of rats at the time of cecal ligation and perforation, a standard model for producing sepsis and a subsequent ARDSlike syndrome.

The results were dramatic - edema and neutrophil accumulation were decreased, and 48 hour mortality was cut in half. Similarly impressive results have been published previously for other therapeutic approaches, but these have failed to translate into effective therapies for ARDS. However, the hope for the present approach lies in the putative mechanism of action of the HSPs in eliciting their cytoprotective effects. The HSPs appear to represent a broad-spectrum defense mechanism, effective in protection against many types of injury. This universality may relate to the fundamental mechanism of action of HSPs, which act as molecular chaperones, maintaining and repairing intracellular proteins (9).

\section{Can HSP therapy be applied in time?}

Translating HSP therapy into clinical practice will have to overcome a number of hurdles. When the stress response was reviewed in the JCI a number of years ago by Minowada and Welch (20), they questioned the applicability of this approach in the treatment of acute clinical conditions because of the many hours required for the stress response to be fully expressed. This criticism certainly rings true with respect to sepsis/ARDS. A number of drugs have been effective when given prior to the onset of sepsis in animal models - an approach rarely possible in the clinical setting (21). In the Weiss study, the authors obtained impressive results even though they did not inject the HSP vector prior to cecal ligation and perforation (3). These encouraging results are similar to those in another animal study that demonstrated a decrease in mortality when the heat shock response was initiated immediately after endotoxin administration (22). Unfortunately, in clinical practice, sepsis is diagnosed by the signs and symptoms of the host response to the septic event, which may only be evident hours to days after the inciting event. Thus for most cases of sepsis/ARDS the translation to the bedside for this therapy is unlikely to be as effective as observed in this study. However, there are clinical situations in which patients at high risk for developing a systemic inflammatory response could be treated early. One such example is in relation to the treatment of biotrauma (23) the inflammatory response that can be induced by mechanical ventilation (24). In this situation, timing of vector administration could easily be instituted at the time of intubation, coincident with or just prior to the start of mechanical ventilation.

\section{The lung as a motor of systemic inflammation}

This study also raises a number of interesting issues in relation to the systemic consequences of sepsis. Weiss and colleagues were surprised at the marked decrease in mortality in their study because pulmonary dysfunction is a rare cause of death in human ARDS; patients usually succumb to multiple organ dysfunction syndrome (MODS) instead. How, then, could a therapy specifically directed to the lungs and unlikely to have caused expression of HSP-70 in other organs (3), affect the pathogenesis of MODS? There are a number of possibilities, but one intriguing hypothesis is that the lung plays a more prominent role in the development of non-pulmonary organ dysfunction in sepsis/ARDS than previously had been thought. As an active metabolic organ, producing a host of cytokines and other mediators, the lung is well placed to affect the development of MODS. The lung also harbors the majority of circulating neutrophils and is subjected to vascular shear forces, and to mechanical forces with each breath. Finally, virtually the entire systemic circulation passes through the lung. In this context any disease process which increases alveolar-capillary permeability in an inflamed lung could lead to translocation of mediators from the lung into the systemic circulation. These mediators may then lead to the development of non-pulmonary organ dysfunction. A similar hypothesis has been proposed for the action of mechanical ventilation in patients with ARDS (25), but in the study by Weiss et al., the animals were not mechanically ventilated.

If the lung is indeed more than an innocent bystander in MODS, HSP therapy directed to this organ might prove broadly useful in the clinical management of sepsis. To date there has been only one therapeutic option that has been proven in a large scale trial to be effective in the treatment of sepsis (26). If the lung represents a motor of systemic inflammation, as has previously been proposed for the gut (27), then, in addition to systemic therapy, therapies of sepsis should 
focus on attacking both pulmonary inflammation and increased alveolarcapillary permeability, whether it be by harnessing the heat shock response, as demonstrated by Weiss and colleagues, or by other innovative approaches.

1. Ritossa, F. 1962. A new puffing pattern induced by temperature shock and DNP in Drosophila. Experientia. 18:571-573.

2. Ashbaugh, D.G., Bigelow, D.B., Petty, T.L., and Levine, B.E. 1967. Acute respiratory distress in adults. Lancet. 2:319-323.

3. Weiss, Y.G., Maloyan, A., Tazelaar, J., Raj, N., and Deutschman, C.S. 2002. Adenoviral transfer of HSP-70 into pulmonary epithelium ameliorates experimental acute respiratory distress syndrome. J. Clin. Invest. 110:801-806. doi:10.1172/ JCI200215888.

4. Tissières, A., Mitchell, H.K., and Tracy, U.M. 1974 Protein synthesis in salivary glands of Drosophila melanogaster: relation to chromosome puffs. J. Mol. Biol. 85:389-398.

5. Lindquist, S., and Craig, E.A. 1988. The heatshock proteins. Annu. Rev. Genet. 22:631-677.

6. Welch, W.J. 1992. Mammalian stress response: cell physiology, structure/function of stress proteins, and implications for medicine and disease. Physiol. Rev. 72:1063-1081.

7. Welch, W.J. 1993. How cells respond to stress. Sci. Am. 268:56-64.

8. Riabowol, K.T., Mizzen, L.A., and Welch, W.J. 1988. Heat shock is lethal to fibroblasts microin- jected with antibodies against hsp70. Science. 242:433-436.

9. Hartl, F.U., and Hayer-Hartl, M. 2002. Molecular chaperones in the cytosol: from nascent chain to folded protein. Science. 295:1852-1858.

10. De Maio, A. 1999. Heat shock proteins: facts, thoughts, and dreams. Shock. 11:1-12.

11. Ware, L.B., and Matthay, M.A. 2000. The acute respiratory distress syndrome. N. Engl. J. Med. 342:1334-1349.

12. Milberg, J.A., Davis, D.R., Steinberg, K.P., and Hudson, L.D. 1995. Improved survival of patients with acute respiratory distress syndrome (ARDS): 1983-1993. JAMA. 273:306-309.

13. dos Santos, C.C., Chant, C., and Slutsky, A.S 2002. Pharmacotherapy of acute respiratory distress syndrome. Expert. Opin. Pharmacother. 3:875-888.

14.2000. Ventilation with lower tidal volumes as compared with traditional tidal volumes for acute lung injury and the acute respiratory distress syndrome. The Acute Respiratory Distress Syndrome Network. N. Engl. J. Med. 342:1301-1308.

15. Wong, H.R. 1998. Potential protective role of the heat shock response in sepsis. New Horiz. 6:194-200.

16. Wong, H.R., and Wispe, J.R. 1997. The stress response and the lung. Am. J. Physiol. 273:L1-L9.

17. Ribeiro, S.P., Villar, J., Downey, G.P., Edelson, J.D., and Slutsky, A.S. 1994. Sodium arsenite induces heat shock protein-72 kilodalton expression in the lungs and protects rats against sepsis. Crit Care Med. 22:922-929.

18. Villar, J., et al. 1994. Induction of the heat shock response reduces mortality rate and organ dam- age in a sepsis-induced acute lung injury model. Crit. Care Med. 22:914-921.

19. Villar, J., Edelson, J.D., Post, M., Mullen, J.B., and Slutsky, A.S. 1993. Induction of heat stress proteins is associated with decreased mortality in an animal model of acute lung injury. Am. Rev. Respir. Dis. 147:177-181.

20. Minowada, G., and Welch, W.J. 1995. Clinical implications of the stress response. J. Clin. Invest. 95:3-12.

21. Crowther, M.A., and Marshall, J.C. 2001. Continuing challenges of sepsis research. JAMA. 286:1894-1896.

22. Chu, E.K., Ribeiro, S.P., and Slutsky, A.S. 1997. Heat stress increases survival rates in lipopoly saccharide-stimulated rats. Crit. Care Med. 25:1727-1732.

23. Tremblay, L.N., and Slutsky, A.S. 1998. Ventilatorinduced injury: from barotrauma to biotrauma. Proc. Assoc. Am. Physicians. 110:482-488.

24. Tremblay, L., Valenza, F., Ribeiro, S.P., Li, J., and Slutsky, A.S. 1997. Injurious ventilatory strategies increase cytokines and c-fos m-RNA expression in an isolated rat lung model. J. Clin. Invest. 99:944-952.

25. Slutsky, A.S., and Tremblay, L.N. 1998. Multiple system organ failure. Is mechanical ventilation a contributing factor? Am. J. Respir. Crit. Care Med. 157:1721-1725.

26. Bernard, G.R., et al. 2001. Efficacy and safety of recombinant human activated protein $\mathrm{C}$ for severe sepsis. N. Engl. J. Med. 344:699-709.

27. Marshall, J.C., and Nathens, A.B. 1996. The gut in critical illness: evidence from human studies. Shock. 6(Suppl. 1):S10-S16. 